\title{
Efecto del estrés en la adquisición y consolidación de la memoria de reconocimiento de objetos en ratas
}

\author{
María R. González-López, Norma L. García-Saldívar, José C. Arriaga-Ramírez y Sara E. Cruz-Morales* \\ Psicofarmacología, Unidad de Investigación Interdisciplinaria en Ciencias de la Salud y Educación (UIICSE), FES-Iztacala, Universidad Nacional \\ Autónoma de México, Tlalnepantla. Ciudad de México, México
}

\begin{abstract}
Resumen
Introducción: Muchas tareas utilizadas en la evaluación del aprendizaje y memoria tienen componentes estresantes. Esto dificulta la interpretación de resultados de los efectos del estrés sobre la mejoría, el deterioro o la ausencia de efecto en la memoria. Se considera que la tarea de reconocimiento de objetos (RO) no contiene estímulos estresantes, por lo que puede ser útil en el estudio del efecto del estrés sobre la memoria. Objetivo: Evaluar el efecto del estrés por restricción (R) (15 min) sobre la adquisición y consolidación de la memoria en RO. Adicionalmente se evaluó la posible activación del eje hipotálamo pituitario adrenal como consecuencia del entrenamiento en RO. Método: Ratas machos Wistar asignadas a cuatro grupos ejecutaron la tarea de $R O$ : dos con $R$ pre o posentrenamiento $(R+R O, R O+R)$, otro recibió corticosterona (C) intraperitonealmente preentrenamiento $(C+R O)$ y uno solo $R O$. Al concluir la tarea, fueron sacrificados inmediatamente y se cuantificó la $C$ plasmática por ensayo por inmunoabsorción ligado a enzimas (ELISA). Para descartar el efecto estresor del entrenamiento en RO, se midió la $C$ en cuatro grupos: uno intacto (I), dos entrenados en RO sacrificados 0 o 24 h después (ERO-0 y ERO-24) y otro restringido $15 \mathrm{~min}$ (R15), sacrificado inmediatamente después. Resultados: La $R$ deterioró la adquisición en los grupos $R+R O$ y $R O+R$; la concentración de $C$ aumentó en $R+R O$ y $C+R O$. En los grupos ERO-0 y $R 15$ aumentó la concentración de $C$ plasmática. Conclusiones: La $R$ deterioró la adquisición en RO. El entrenamiento en RO aumentó la concentración de $C$ en plasma similar a R15, indicando la naturaleza estresante de la tarea. Es probable que el deterioro de memoria en el grupo $R+R O$ se deba a la suma de la elevación de la $C$ inducida por la $R$ y a los cambios inducidos por el entrenamiento en RO. La inyección de C no modificó la memoria.
\end{abstract}

Palabras clave: Memoria de reconocimiento. Adquisición. Estrés. Corticosterona. Restricción.

\section{The effect of stress on acquisition and consolidation of object recognition memory in rats}

\begin{abstract}
Introduction: Many tasks used in the evaluation of learning and memory have stressful components. This makes it difficult to interpret the effects of stress on improvement, impairment or no effect on memory. It is considered that the object recognition task (RO) contain no stressful stimuli; therefore, it can be useful in the study of the effect of stress on memory. Objective: To evaluate the effect of restraint stress $(R)(15 \mathrm{~min})$ on acquisition and consolidation of memory in RO. In
\end{abstract}

\section{Correspondence:}

*Sara Eugenia Cruz-Morales

Psicofarmacología, UIICSE, FES-Iztacala

Universidad Nacional Autónoma de México

Av. de los Barrios, 1

Fecha de recepción: 4-06-2018

Fecha de aceptación: 30-11-2018

DOI: 10.24875/RMN.M19000045
Disponible en internet: 18-06-2019 Rev Mex Neuroci. 2019;20(3):141-148 www.revmexneurociencia.com 
addition, the possible activation of the hypothalamic-pituitary-adrenal axis was evaluated as a consequence of the training in RO. Methods: Male Wistar rats assigned to four groups carried of the task of $R O$ : two with $R$ pre or post-training $(R+R O$, $R O+R)$, another received corticosterone $(C)$ intraperitoneally pre-training $(C+R O)$ and one $R O$ only $(R O)$. Thereafter, they were immediately sacrificed and plasma $C$ was quantified. To exclude the stressor effect of training in $R O, C$ was measured in four groups: one intact (I), two trained in RO sacrificed 0 or $24 \mathrm{~h}$ later (ERO-O and ERO-24) and another restricted 15 min (R15), sacrificed immediately after. Results: The $R$ impaired the acquisition in the groups $R+R O$ and $R O+R$; the concentration of $C$ increased in $R+R O$ and $C+R O$. In ERO-O and R15, the plasma $C$ increased. Conclusions: The pre-training $R$ impaired memory. Training in $R O$ increased the plasma $C$ similar to $R 15$ indicating the stressful nature of the task. The $C$ injection did not modify memory.

Key words: Recognition memory. Acquisition. Stress. Corticosterone. Restriction.

\section{Introducción}

El estrés facilita, deteriora o no influye en las etapas de la memoria en ratas $^{1-3} y$ humanos ${ }^{4}$, su efecto interviene en los circuitos cerebrales involucrados ${ }^{5,6}$. La respuesta depende de las características del estresor ${ }^{7}$, la duración y naturaleza de la tarea ${ }^{8-10}$, y factores fisiológicos del sujeto que influyen en las respuestas neuroendocrinas y conductuales ${ }^{11}$.

El estrés provoca liberación de corticosterona (C) por las glándulas suprarrenales ${ }^{12,13}$. Niveles de estrés alto o bajo deterioran la memoria y un nivel moderado la mejora $^{14}$. La administración de C puede mejorar ${ }^{15} 0$ deteriorar la memoria ${ }^{14,16,17}$, lo que depende de la dosis, la experiencia emocional o la fase de la memoria estudiada ${ }^{18,19}$.

La naturaleza de la tarea es importante. En el estudio de la memoria de procedimiento hemos utilizado dos tareas: la de evitación inhibitoria (EI) y el laberinto elevado en T (LET). En la El, la administración del choque provoca que los animales aprendan a evitar el estímulo aversivo durante el entrenamiento. Con esta tarea se han estudiado diferentes estructuras cerebrales y la participación de sistemas de neurotransmisión como el colinérgico ${ }^{20,21}$. Anticolinérgicos como la escopolamina administrada intraperitonealmente ${ }^{22} 0$ intraestriatal indujeron amnesia ${ }^{23}$. La escopolamina posentrenamiento no produjo amnesia en intensidades de 1 y $1.5 \mathrm{~mA}$; con intensidades de 2 y $2.5 \mathrm{~mA}$ produjo amnesia ${ }^{22}$ y altas intensidades, 2.8 y $3.0 \mathrm{~mA}$, no deterioraron la memoria. Se ha propuesto que con altas intensidades de choque, la actividad colinérgica no es necesaria para la consolidación ${ }^{24}$. Estudios realizados con agonistas GABAérgicos demostraron quela administración de muscimol indujo amnesia ${ }^{25}$ y potenció la amnesia inducida por escopolamina, con intensidades bajas ${ }^{26}$.

Los componentes aversivos de la tarea con el LET fueron comparados con estresores como la restricción de movimiento $(R)^{27}$, estresor traumático para los roedores ${ }^{28}$ que activa el eje hipotálamo pituitario adrenal (HPA $)^{27}$. La exposición pre entrenamiento a $60^{29} \mathrm{o}$ 15 min de $\mathrm{R}^{30}$ deterioraron la memoria pero no la exposición posentrenamiento ${ }^{31}$. Estas dos tareas son consideradas aversivas por la presencia de los espacios abiertos y el choque, componentes considerados estresores intrínsecos ${ }^{32}$. La exposición al entrenamiento en LET activa el eje HPA ${ }^{33}$ y el choque en El aumenta la concentración de $\mathrm{C}^{30}$, por lo que los resultados podrían interpretarse como la suma de los estresores intrínsecos más el estresor extrínseco con efecto en el entrenamiento.

La tarea de reconocimiento de objetos $(\mathrm{RO})$ evalúa la memoria declarativa mediante la preferencia por la novedad $^{34,35}$. Tiene la ventaja de que los ensayos pueden ser modificados para examinar diferentes componentes de la memoria, el efecto del estrés y la liberación de hormonas. Se ha propuesto que carece de estímulos aversivos por no requerir de privación de agua y comida ${ }^{36,37}$. El objetivo de este trabajo fue evaluar en una tarea de memoria de reconocimiento de objetos, el efecto del estrés por R (15 min) sobre la adquisición y consolidación de la memoria y estudiar la posible activación del eje HPA como consecuencia del entrenamiento de la tarea.

\section{Método}

\section{Sujetos}

Los experimentos fueron realizados siguiendo las normas establecidas por las guías de conducta ética en el cuidado y uso de animales no humanos de la Society for Neuroscience. Se utilizaron ratas Wistar machos adultos ingenuos experimentalmente (250-270 g) provenientes del bioterio de la Universidad Nacional Autónoma de México, FES-Iztacala. Los sujetos fueron alojados en el bioterio, con agua y alimento ad libitum, con un ciclo de luz-oscuridad (8:00 a 
20:00 h), donde permanecieron en estas condiciones durante cinco días previos al experimento. Al inicio de los experimentos los sujetos $(n=56)$ se asignaron aleatoriamente a diferentes grupos. Los grupos experimentales que ejecutaron la tarea de memoria de reconocimiento recibieron una sesión de habituación de 10 min en la caja de reconocimiento libre de objetos un día previo al entrenamiento.

\section{Fármaco}

Corticosterona en polvo C2505 (Sigma-Aldrich).

\section{Aparatos}

- Restrictores. Se emplearon contenedores de acrílico cilíndricos (Plas-Labs, Inc ${ }^{\mathrm{TM}}$ ) con hoyos ajustables a la talla del sujeto experimental. Los sujetos fueron restringidos en un cuarto con luz natural durante 15 min.

- Lector de placas. Para medir la concentración de C se utilizó un lector de placas (H Reader 1 de HLAB), con filtro de 405 a $630 \mathrm{~nm}$ y una resolución de 0.0001 de absorbancia.

- Cámara de reconocimiento de objetos. Se empleó una cámara construida de PVC blanco $(100 \times 100 \times$ $50 \mathrm{~cm}$ ), con el piso cuadriculado $(10 \mathrm{~cm})$ que permitió colocar los objetos siempre a la misma distancia. Una cámara de circuito cerrado (AVTECH de 4 canales AVTECH AVC792DBKIT) grabó los ensayos de los sujetos con el fin de visualizar y hacer mediciones mediante un software para colección y análisis de datos Observer ${ }^{38}$.

- Objetos familiares y novedosos. Se utilizaron legos infantiles de plástico de color azul para los objetos familiares (Fig. 1A) variando el color y la forma para el objeto novedoso (Fig. 1B). La parte interna de los legos se saturaron de acrílico dental con el fin de que pesaran y la rata no los moviera.

- Tarea de RO. Las sesiones experimentales se realizaron entre las 8:00 y 11:00 h. En los ensayos de entrenamiento se colocó al sujeto en la cámara con dos objetos idénticos ( $F 1$ y F2) y se les permitió explorarlos en una prueba de familiarización durante 5 min. La exploración se definió como el acercamiento de los sujetos dirigiendo la nariz hacia los objetos en un rango de $2 \mathrm{~cm} 0$ cuando olfateaban. Dar la vuelta a/o sentarse sobre el objeto no se consideraron una conducta de exploración ${ }^{39,40}$. El criterio de inclusión en el grupo fue que los sujetos exploraran por lo menos $10 \mathrm{~s}$ durante los ensayos de

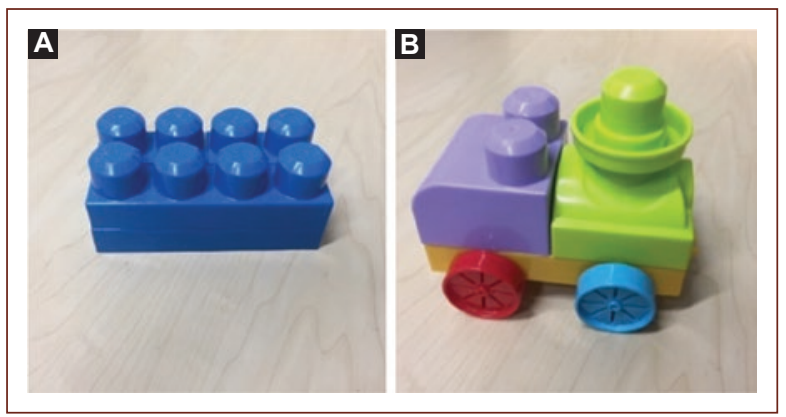

Figura 1. Objetos utilizados en la tarea de reconocimiento de objetos. A: objeto familiar (OF). B: objeto novedoso (ON).

entrenamiento solo en los grupos que no hubieran sido expuestos a tratamiento antes del entrenamiento. En los grupos con tratamiento previo al entrenamiento, se respetaron los tiempos de exploración como efecto de este.

En el ensayo de prueba después de un periodo de retardo de $24 \mathrm{~h}$ posteriores al entrenamiento se expuso a los sujetos a un objeto familiar (OF) y a un objeto novedoso (ON) durante 5 min.

Se registró el tiempo de exploración al objeto novedoso (TN) y al objeto familiar (TF) y se calculó un índice de discriminación para cada sujeto que se expresó como ID = 100 * (TN $-\mathrm{TF}) /(\mathrm{TN}+\mathrm{TF})$, donde TF = tiempo dedicado a explorar al objeto familiar y $\mathrm{TN}=$ tiempo dedicado a explorar al objeto novedoso. El índice de discriminación (ID) mayor a cero, muestra que los animales exploraron más al ON que al OF, por lo que se considera buena memoria, mientras que una proporción cercana a cero indica que los animales exploraron el mismo tiempo a los dos objetos, lo que se considera deterioro de la memoria ${ }^{35,36}$.

\section{Cuantificación de la corticosterona}

Al concluir las sesiones experimentales, los sujetos se sacrificaron por decapitación entre las 8:00 y 11:00 $\mathrm{h}$, se obtuvieron muestras de sangre del tronco $(3 \mathrm{ml})$ que fueron centrifugadas a 3,000 rpm. Inmediatamente después el suero se mantuvo a $-20^{\circ} \mathrm{C}$ hasta ser procesado, por medio de una prueba de enlace de inmunoensayo (Assay Designs, Catálogo n. 900-097 EV, Ann Arbor, Michigan, EE.UU.); los resultados analíticos son expresados en $\mathrm{ng} / \mathrm{ml}$. El límite bajo de detección fue de $0.027 \mathrm{ng} / \mathrm{ml}$ y el porcentaje inter e intraensayo tuvo un coeficiente de variación de 5.3 y 9.87 respectivamente. 


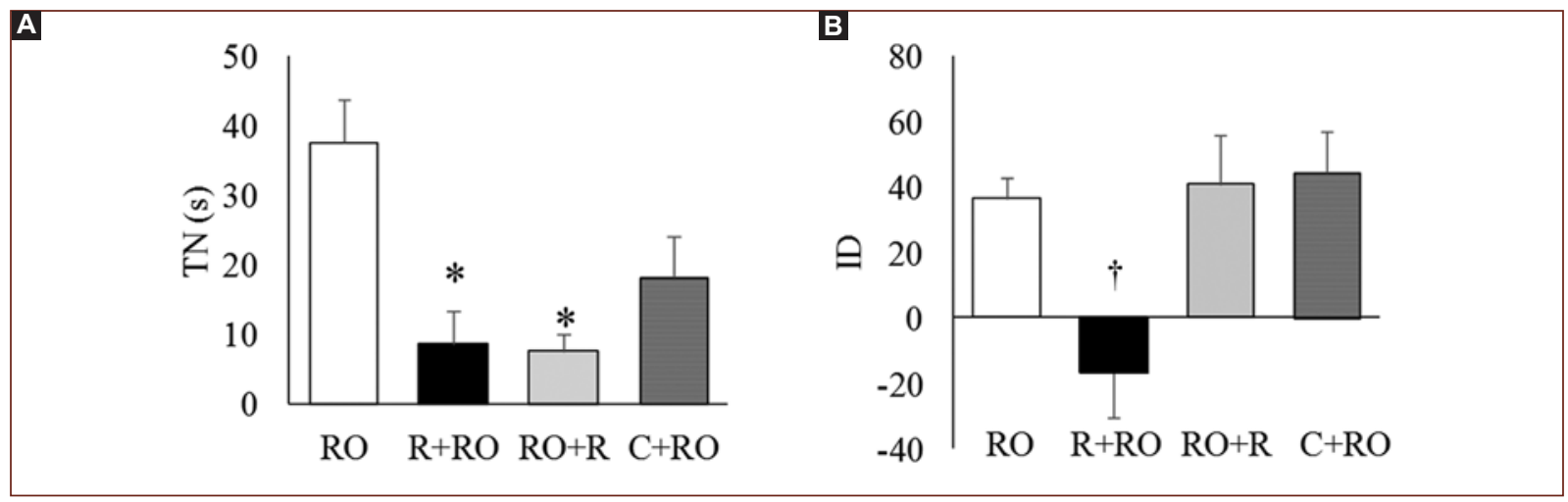

Figura 2. Gráficas que muestran a sujetos expuestos a restricción $(R)$ antes o después del entrenamiento $(R+R 0, R 0+R)$ 0 de la administración de corticosterona $(5 \mathrm{mg} / \mathrm{kg})(\mathrm{C}+\mathrm{RO})$. A: media $( \pm \mathrm{EEM})$ del tiempo de exploración al objeto novedoso (TN). B: media ( \pm EEM) del índice de discriminación (ID) en la tarea de RO.

${ }^{*} \mathrm{p}<0.01$.

tp $<0.05$.

\section{Procedimiento}

Se utilizaron 32 sujetos asignados a cuatro grupos $(\mathrm{n}=8)$ que recibieron los siguientes tratamientos: un grupo que ejecutó la tarea de RO; dos grupos que realizaron el entrenamiento de la tarea de $\mathrm{RO}$ con la diferencia que uno fue sometido a $\mathrm{R}$ antes del entrenamiento (estrés en la fase de adquisición) $(R+R O)$ y otro fue entrenado e inmediatamente después sometido a $R$ (estrés en la fase de consolidación) $(R O+R)$; a un cuarto grupo se le administró $5 \mathrm{mg} / \mathrm{kg}$ intraperitoneal (ip) antes del entrenamiento para simular la $\mathrm{C}$ liberada por la exposición a $\mathrm{R}(\mathrm{C}+\mathrm{RO})$. La prueba de memoria se realizó en todos los grupos $24 \mathrm{~h}$ después del entrenamiento. Los animales fueron sacrificados después de la prueba.

Adicionalmente se evaluó la liberación de $\mathrm{C}$ en plasma después de exponer a los sujetos al entrenamiento de RO para descartar que la sola exposición al entrenamiento active el eje HPA como ocurre en otras tareas $^{33}$. Se asignaron 24 ratas a cuatro grupos $(n=6)$, que recibieron los siguientes tratamientos: un grupo intacto (I), sin ningún tratamiento, un grupo expuesto a 15 min de R sacrificado inmediatamente después (R15); otros dos grupos expuestos solo al entrenamiento en RO sacrificados 0 o $24 \mathrm{~h}$ después (ERO-0 y ERO-24).

\section{Estadística}

Dado que se confirmó la homogeneidad de las varianzas, para analizar los efectos en el total del TN y el ID entre grupos, se empleó un análisis de varianza de una vía (ANOVA), cuando hubo diferencias se utilizó una prueba post hoc de Honestly Significant Difference (HSD) de Tukey. Para analizar las diferencias entre TN y TF de cada grupo se realizaron pruebas de t de Student pareada. Como los datos de la concentración de $\mathrm{C}$ no presentaron homogeneidad de varianzas, se realizó una prueba de Kruskal-Wallis seguida de una prueba $U$ de Mann-Whitney. Todos los análisis estadísticos se realizaron mediante el software STATISTICA ${ }^{\circledR}$ (versión 7).

\section{Resultados}

En la figura 2A se muestra el tiempo de exploración al objeto novedoso de los diferentes tratamientos. Se observaron diferencias significativas $(F[3,28]=7.60$; $p<0.001)$ entre los grupos. Los sujetos de los grupos $\mathrm{R}+\mathrm{RO}$ y $\mathrm{RO}+\mathrm{R}$ permanecieron menos tiempo explorando el ON $(p<0.01)$ respecto al que ejecutó la tarea sin R. La administración de $C$ antes del entrenamiento no tuvo efecto. En la figura 2B se muestra el ID, se encontraron diferencias significativas entre los grupos $(F[3,28]=5.37 ; p<0.01)$; el grupo $\mathrm{R}+\mathrm{RO}$ difirió de todos los grupos $(p<0.05)$, mostrando una menor discriminación al $\mathrm{ON}$. Los grupos de $\mathrm{RO}+\mathrm{R}$ y de $\mathrm{C}+\mathrm{RO}$ no presentaron diferencias respecto a RO.

La prueba $t$ de Student mostró que el grupo que ejecutó la tarea de RO sin ningún tratamiento exploró más el $\mathrm{ON}(\mathrm{t}[7]=3.53 ; p<0.01)$. Hubo menor exploración de los objetos en los grupos que fueron sometidos a $\mathrm{R}$ o que recibieron $\mathrm{C}$. No obstante, se observó que en el grupo de $\mathrm{R}+\mathrm{RO}$ el TN y el TF fueron similares ( $t[7]=0.2 ; p=0.843$ ). La exploración al ON fue mayor 
en los grupos que recibieron $\mathrm{R}$ después del entrenamiento o C (t $[7=2.93 ; p<0.05)$ (Fig. 3).

La concentración de $\mathrm{C}$ en plasma presentó diferencias significativas entre los grupos $(H[3, n=31]=15.727$; $p<0.001$ ). Los sujetos que fueron sometidos a $R$ o a la administración de $\mathrm{C}$ antes del entrenamiento mostraron mayor concentración de $C$ plasmática $(p<0.05)$, mientras que en el grupo expuesto a $\mathrm{R}$ posentrenamiento la concentración fue parecida al grupo RO (Fig. 4).

Finalmente, la figura 5 muestra la concentración de $C$ en plasma de los grupos expuestos al entrenamiento en RO o a la R $15 \mathrm{~min}$; se observaron diferencias $(H[4, n=30]=13.21 ; p<0.05)$. Los grupos que recibieron $\mathrm{R} o$ el entrenamiento en $\mathrm{RO}$ y que fueron sacrificados inmediatamente después mostraron mayor concentración de $C$ respecto al grupo intacto $(p<0.05)$, no así los que fueron sacrificados a las $24 \mathrm{~h}$, lo que denota que el entrenamiento en la tarea de RO estimula al eje HPA de forma similar a la exposición a $R$ cuando se registra inmediatamente.

\section{Discusión}

Nuestros resultados demostraron deterioro en la adquisición de la memoria de reconocimiento de los sujetos expuestos a $R$ antes del entrenamiento, evaluados en la fase de prueba 24 h después, según el TN y el ID, parámetros empleados en esta prueba. Lo anterior es consistente con lo reportado en otros estudios $^{41}$ donde demostraron que el efecto de $\mathrm{R}$ en $\mathrm{RO}$ provocó deterioro cuando se aplicó antes del entrenamiento y la fase de prueba se realizó en intervalos de $3 \mathrm{~h}$, pero no cuando se realizó la prueba $5 \mathrm{~min}$ después. Efectos similares se encontraron en este laboratorio con la exposición a 15 min de $\mathrm{R}$ preentrenamiento y una demora de fase de prueba de dos horas que también provocó deterioro de la memoria de $\mathrm{RO}^{42}$. Como puede observarse para esta tarea las demoras entre la fase de entrenamiento y la prueba son determinantes para poder evaluar el ID y el $\mathrm{TN}^{43}$, ya que los intervalos largos pueden influir en la reducción del ID ${ }^{39}$; sin embargo, con esta tarea se pudieron observar efectos del estrés $24 \mathrm{~h}$ después. La exposición a $\mathrm{R}$ antes del entrenamiento se ha observado en otras tareas como LET, ya que 15 o 60 min de R ocasionaron deterioro de la memoria cuando el entrenamiento se realizó inmediatamente después ${ }^{29,30}$

En la consolidación de RO no se observó un efecto significativo en el ID, lo que implica que los sujetos discriminaron en forma similar al grupo que realizó la tarea sin R. Cuando se compararon TN y TF durante

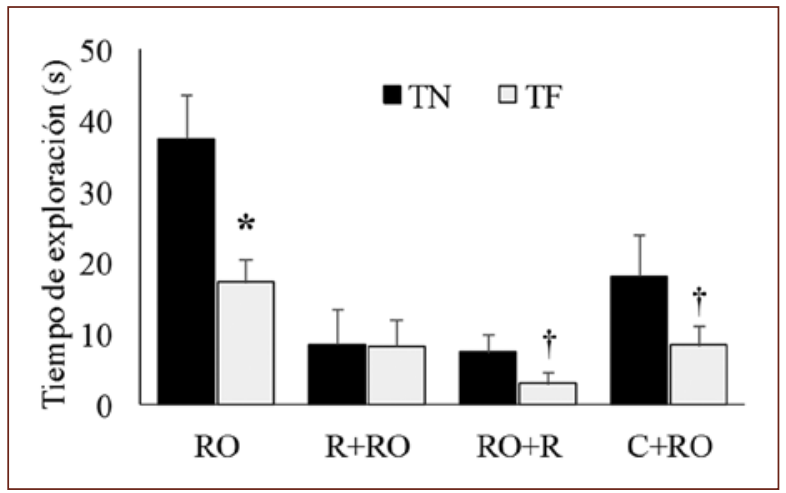

Figura 3. Medias ( \pm EEM) del tiempo de exploración al objeto novedoso (TN) o al objeto familiar (TF) en sujetos sin restricción (RO), expuestos a restricción preentrenamiento o posentrenamiento $(R+R 0, R 0+R) 0$ $5 \mathrm{mg} / \mathrm{kg}$ de corticosterona $(\mathrm{C})$ preentrenamiento $(\mathrm{C}+\mathrm{RO})$ que realizaron la tarea de $\mathrm{R} 0$.

${ }^{*} p<0.01$ frente a TN de su grupo.

tp $<0.05$ frente a TN de su grupo.

Efecto del estrés sobre la concentración de corticosterona en plasma

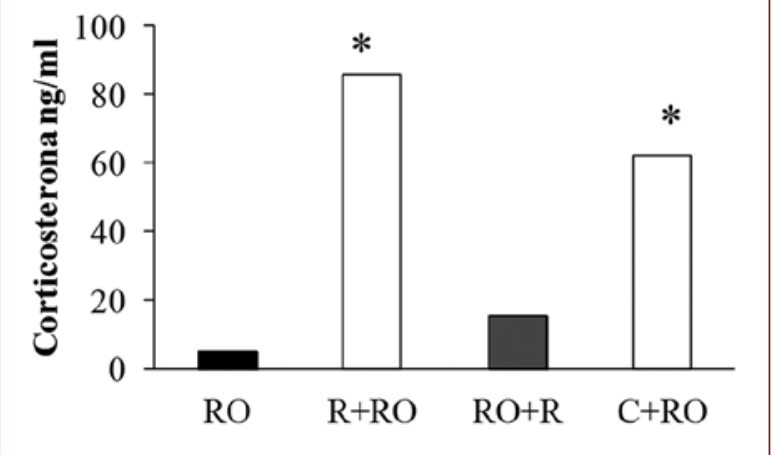

Figura 4. Medianas de la concentración de corticosterona (C) en plasma de sujetos que realizaron la tarea de reconocimiento de objetos sin restricción (RO), expuestos a restricción preentrenamiento 0 posentrenamiento $(\mathrm{R}+\mathrm{RO}, \mathrm{R} 0+\mathrm{R})$ o $5 \mathrm{mg} / \mathrm{kg}$ de $\mathrm{C}$ preentrenamiento $(\mathrm{C}+\mathrm{R} 0)$. ${ }^{*} p<0.05$ frente al grupo de RO.

la prueba, los sujetos del grupo $\mathrm{RO}+\mathrm{R}$ visitaron más el $\mathrm{ON}$, pero exploraron menos que el grupo sin $\mathrm{R}$, esto puede estar relacionado con un efecto de ansiedad inducido por la caja utilizada para desarrollar la tarea, ya que los sujetos muestran menor tiempo de exploración. El efecto de $R$ sobre la consolidación evaluado con el ID, coincide con los resultados obtenidos en otros estudios con 60 min de $R$ precedidos por el entrenamiento en $\mathrm{RO}$ en ratones? ${ }^{2}$. 
Efecto estresor del entrenamiento en RO y de la exposición a R

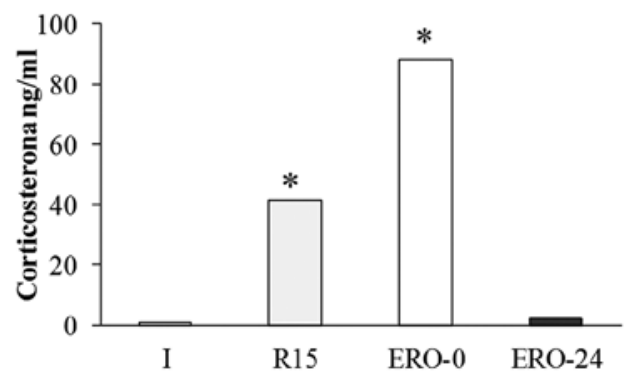

Figura 5. Medianas de la concentración de corticosterona (C) en plasma de ratas sometidas a restricción (R15) 0 entrenamiento en reconocimiento de objetos ( $R O)$ sin restricción (I) (ERO-0 y ERO-24), sacrificadas a las $0024 \mathrm{~h}$. ${ }^{*} p<0.05$ frente a $\mathrm{I}$.

Los sujetos a los que se les administró $C$ preentrenamiento no presentaron deterioro de RO; efectos similares se han reportado en una tarea de alternación espacial ${ }^{18}$. La concentración en plasma medida $24 \mathrm{~h}$ después en los grupos que realizaron la tarea de RO aumentó solo en los grupos que fueron expuestos a $\mathrm{R} \circ \mathrm{C}$ preentrenamiento. Esto implica que la sola elevación de $\mathrm{C}$ no es suficiente para generar cambios en la memoria de $\mathrm{RO}$ y aunque la concentración es similar parece que el efecto en la memoria dependió del momento de la presentación de la R.

Hay tareas que por sus características aversivas generan activación del eje HPA durante el entrenamiento ${ }^{31,33}$. En diversos estudios que utilizan la tarea de $\mathrm{RO}$ para evaluar la memoria emplean una caja cuadrada o rectangular, esta misma tarea se ha utilizado como campo abierto para evaluar la respuesta de ansiedad $^{44,45}$.

El efecto del estrés por $\mathrm{R}$ en la adquisición de la memoria en RO se puede explicar por el estado emocional del animal durante el entrenamiento ${ }^{46}$. Existen estudios que relacionan la presentación de estresores como la restricción, cercanos a la fase de la adquisición con la expresión diferencial de receptores a glucocorticoides bajos y receptores a mineralocorticoides altos en hipocampo, que pueden generar alteración en el procesamiento de la información ${ }^{47}$. En nuestros resultados es probable que el efecto de la $\mathrm{R}$ se sume al estrés producido durante el entrenamiento en RO y que ambos modifiquen la codificación de la información emocional en las estructuras cerebrales involucradas durante la adquisición ${ }^{48}$.
Los resultados obtenidos en el ID y el TN sugieren que para que ocurra deterioro de memoria se requiere que los sujetos sean expuestos a la suma de factores estresantes; en este caso el estrés extrínseco producido por la $\mathrm{R}$ e intrínseco ocasionado por el entrenamiento en RO. En estas condiciones es probable que se modifique el estado emocional durante el entrenamiento en RO y sensibilice el eje HPA, de tal forma que durante la exposición en la sesión de prueba de RO se favorezca la liberación de $C^{49}$.

La cercanía entre ambos estresores es fundamental, porque en otros estudios, cuando se administraron 60 o 75 min de $R$ antes del entrenamiento en RO, no se vio efecto en la memoria, ${ }^{2}$ tampoco 60 min de R aplicados $24 \mathrm{~h}$ antes en la tarea de alternación espacial tuvieron efecto ${ }^{18}$.

En el presente estudio se observó mayo concentración de $\mathrm{C}$ en los animales evaluados inmediatamente después del entrenamiento, respecto a los evaluados $24 \mathrm{~h}$ después. Los resultados muestran que el entrenamiento en la tarea de RO activa la respuesta de estrés en los periodos inmediatos a la tarea y se comporta como un estresor que se restablece por retroalimentación negativa, ya que a las $24 \mathrm{~h}$ la concentración de $C$ vuelve a condiciones basales.

Aunque los sujetos fueron habituados a la cámara $24 \mathrm{~h}$ antes del entrenamiento durante $10 \mathrm{~min}$, la reexposición a la cámara de RO provocó activación del eje HPA el día del entrenamiento. El animal en el entrenamiento se enfrenta a dos objetos similares que son extraños o novedosos para él, la liberación de $C$ es un índice importante de estrés en ratas y sugiere que el confinamiento en un ambiente nuevo es estresante ${ }^{50}$. La respuesta del sujeto a una cámara que durante la habituación estaba vacía y durante el entrenamiento se expone a dos objetos, implica un nuevo contexto, y es probable que esta exposición le provoque activación de la atención, la ansiedad y de factores relacionados con la respuesta de estrés como la liberación de glucocorticoides ${ }^{41,42}$.

Ennaceur ${ }^{39}$ considera que la tarea de RO se realiza en bajas condiciones de estrés y otros autores señalan que la habituación al contexto experimental reduce la respuesta de agitación ${ }^{51}$. Sin embargo, los resultados de nuestro estudio indican lo contrario, ya que la situación emocional del sujeto persiste en el entrenamiento de la tarea de RO, lo que permite disociar el estrés intrínseco, inherente a la tarea o situación de aprendizaje ${ }^{32}$, ya que la exposición solo al entrenamiento es comparable al efecto ocasionado por la R. 


\section{Conclusiones}

Los resultados obtenidos en este estudio sugieren que 15 min de $R$ antes del entrenamiento en $\mathrm{RO}$ deterioran la memoria declarativa a las $24 \mathrm{~h}$ y desencadenan la liberación de $\mathrm{C}$ en plasma. La inyección de $\mathrm{C}$ pre entrenamiento en dosis de $5 \mathrm{mg} / \mathrm{kg}$ reproduce las concentraciones plasmáticas bajo estrés, pero no tiene efecto sobre la memoria. Se demostró el efecto estresor del entrenamiento de la tarea de RO.

\section{Financiamiento}

El estudio se realizó con el apoyo del Programa de Apoyo a Proyectos de Investigación e Innovación Tecnológica (PAPIIT) de la Universidad Nacional Autónoma de México, con el número de proyecto IN306216 otorgado a Sara Eugenia Cruz Morales.

\section{Conflicto de intereses}

Los autores declaran no tener conflicto de intereses.

\section{Bibliografía}

1. Bisaz R, Conboy L, Sandi C. Learning under stress: A role for the neural cell adhesion molecule NCAM. Neurobiol Learn Mem. 2009;91:333-42.

2. Li S, Fan YX, Wang W, Tang YY. Effects of acute restraint stress on different components of memory as assessed by object-recognition and object-location tasks in mice. Behav Brain Res. 2012;227(1):199-207.

3. Wirth M. Hormones, stress, and cognition: The effects of glucocorticoids and oxytocin on memory. Adapt Human Behav Physio. 2015;1(2):177-201.

4. Corbett B, Winberg L, Duarte A. The effect of mild acute stress during memory consolidation on emotional recognition memory. Neurobiol Learn Mem. 2017 145:33-44.

5. Butler RK, Oliver EM, Sharko AC, Parrilla-Carrero J, Kaigler KF, Fadel JR, et al. Activation of corticotrophin releasing factor-containing neurons in the rat central amygdale and bed nucleus of the stria terminalis following exposure to two different anxiogenic stressors. Behav Brain Res. 2016; 304:92-101

6. Ness D, Calabrese P. Stress effects on multiple memory system interactions. Neural Plast. 2016;2016:4932128.

7. Howland JG, Cazakoff BN. Effects of acute stress and GluN2B-containing NMDA receptor antagonism on object and object-place recognition memory. Neurobio Learn Mem. 2010:93:261-7.

8. Cazakoff BN, Johnson KJ, Howland JG. Converging effects of acute stress on spatial and recognition memory in rodents: A review of recent behavioural and pharmacological findings. Pro Neuropsychopharmacol Biol Psychiatry. 2010;34:733-41

9. Joëls M, Baram TZ. The neuro-symphony of stress. Nat Rev Neurosci. 2009;10(6):459-66.

10. Sardari M, Rezayof A, Khodagholi F. Hippocampal signaling pathways are involved in stress-induced impairment of memory formation in rats. Brain Res. 2015;1625:54-63.

11. Joëls M, Sarabdjitsingh RA, Karst $H$. Unraveling the time domains of corticosteroid hormone influences on brain activity: Rapid, slow and chronic modes. Pharmacol Rev. 2012;64:901-38.

12. Herman JP, McKlveen JM, Ghosal S, Kopp B, Wulsin A, Makinson R, et al. Regulation of the hypothalamic-pituitary-adrenocortical stress response. Compr Physiol. 2016;6(2):603-21.

13. Wolf OT. Stress and memory retrieval: mechanisms and consequences. Behav Sci. 2017:14:40-6.

14. Vargas-López V, Torres-Berrio A, González-Martínez L, Múnera A, Lamprea MR. Acute restraint stress and corticosterone transiently disrupts novelty preference in an object recognition task. Behav Brain Res. 2015; 291:60-6.

15. Barsegyan A, Mackenzie SM, Kurose BD, McGaugh JL, Roozendaal B. Glucocorticoids in the prefrontal cortex enhances memory consolidation and impair working memory by a common neural mechanism. Proc Nat Acad Sci U S A. 2010;107(38):16655-60.

16. Atsak P, Guenzel FM, Kantar-Gok D, Zalachoras I, Yargicoglu P, Meijer OC, et al. Glucocorticoids mediate stress-induced impairment of retrieval of stimulus-response memory. Psychoneuroendocrinology. 2016;67:207-15.

17. Roozendaal B. Stress and memory: Opposing effects of glucocorticoids on memory consolidation and memory retrieval. Neurobiol Learn Mem. 2002;78:578-95

18. Sadowski RN, Jackson GR, Wieczorek LA, Gold PE. Effects of stress, corticosterone, and epinephrine administration on learning in place and response tasks. Behav Brain Res. 2009;205:19-25.

19. Yang Ch, Liu J, Chai B, Fang Q, Chai N, Zhao LY, et al. Stress within a restricted time window selectively affects the persistence of long-term memory. PLoS One. 2013;8:e59075.

20. Prado-Alcalá RA, Cruz-Morales SE, López-Miro FA. Differential effects of cholinergic blockade of anterior and posterior caudate nucleus on avoidance behaviors. Neurosci Lett. 1980;18:339-45.

21. Prado-Alcalá RA. Is cholinergic activity of the caudate nucleus involved in memory? Life Sci. 1985;37:2135-42.

22. García-Saldívar NL. Efecto de agonistas y antagonistas GABAérgicos sobre la amnesia inducida por escopolamina, en ratas sometidas a un condicionamiento de evitación pasiva en condiciones de bajo reforzamiento [Tesis de Maestría en Internet]. México: Universidad Nacional Autónoma de México, Programa de Maestría y Doctorado en Psicología; 2002. Disponible en: http://132.248.9.195/pdtestdf/0302175//ndex.html

23. González-López MRA, García-Saldívar NL, Gómez-Romero JG, Arriaga-Ramírez PA, Cruz-Morales SE. Modulación de la actividad GABAérgica del estriado sobre la amnesia inducida por escopolamina. Rev Mex Psicol. 2003;20:283-9.

24. Cruz-Morales SE, Durán-Arévalo M, Díaz del Guante MA, Quirarte G, Prado-Alcalá RA. A threshold for the protective effect of over-reinforced passive avoidance against scopolamine-induced amnesia. Behav Neural Biol. 1992;57:256-9.

25. Veloz-Gómez L. Interacción colinérgica y GABAérgica en una tarea de evitación inhibitoria entrenada con bajas intensidades [Tesis de Licenciatura en Internet]. México: Universidad Nacional Autónoma de México; 1999. Disponible en: http://132.248.9.195/pd1999/271194/Index.html

26. Cruz-Morales SE. Interacción de los sistemas colinérgico y GABAérgico en la consolidación de una respuesta de evitación inhibitoria [Tesis de Doctorado en Internet] México: Programa de Doctorado en Ciencias Biomédicas, Universidad Nacional Autónoma de México; 1992. Disponible en: http://132.248.9.195/pmig2016/0183543/lndex.html

27. García-Saldívar NL, González-López MR, Monroy J, Dominguez R, Cruz-Morales SE. Restriction stress modifies monoamines activity in the prefrontal cortex of rats submitted to an inhibitory avoidance task. Annual Meeting SfN 2014; Sn. Diego, Cal., USA, 628.26. Abstract

28. Armario A, Escorihuela RM, Nadal. Long-term neuroendocrine and behavioural effects of a single exposure to stress in adult animals. Neurosci Biobehav Rev. 2008:32:1121-35.

29. Cruz-Morales SE, García-Saldívar NL, González-López MR, Castillo-Roberto G, Monroy J, Domínguez R. Acute restriction impairs memory in the elevated T-maze (ETM) and modifies serotonergic activity in the dorsolateral striatum. Behav Brain Res. 2008;195:187-91.

30. García-Saldívar NL. Efecto del estrés agudo por restricción sobre la memoria y la ansiedad: participación de la noradrenalina en la CPF y núcleo estriado [Tesis de Doctorado en Internet]. México: Programa de Maestría y Doctorado en Psicología, Universidad Nacional Autónoma de México; 2017. Disponible en: http://132.248.9.195/ptd2017/febrero/070501788/Index.html

31. Favila GP. Efectos de la administración de corticosterona e inducción de estrés por restricción en una prueba de LET sobre la consolidación y recuperación de la memoria [Tesis de Licenciatura en Internet]. México: Facultad de Psicología, Universidad Nacional Autónoma de México; 2015. Disponible en: http://132.248.9.195/ptd/2015/agosto/307075374/ Index.html

32. Sandi C, Pinelo-Nava MT. Stress and memory: Behavioral effects and neurobiological mechanisms. Neural Plast. 2007;2007:78970.

33. Graeff FG, Zangrossi Jr. H. The hypothalamic-pituitary-adrenal axis in anxiety and panic. Psychol Neurosci. 2010;3(1):3-8.

34. Cohen SJ, Stackman Jr RW. Assessing rodent hippocampal involvement in the novel object recognition task: A review. Behav Brain Res. 2015 285:105-17

35. Nava-Mesa MO, Lamprea MR, Múnera A. Divergent short- and long-term effects of acute stress in object recognition memory are mediated by endogenous opioid system activation. Neurobiol Learn Mem. 2013; 106:185-92.

36. Jurado-Berbel P, Costa-Miserachs D, Torras-Garcia M, Coll-Andreu M, Portell-Cortés I. Standard object recognition memory and "what" and "where" components: Improvement by post-training epinephine in highly habituated rats. Behav Brain Res. 2010;207:44-50.

37. Luine, V. Recognition memory task in neuroendocrine research. Behav Brain Res. 2015;285:158-64. 
Rev Mex Neuroci. 2019;20

38. Observer, Software de dominio público del Laboratorio de Psicobiología de la Universidad de Säo Paulo, Brazil.

39. Ennaceur A. One trial object recognition in rats and mice: Methodological and theoretical issues. Behav Brain Res. 2010;215:244-54.

40. Zhao X Li Y, Peng T, Seese RR, Wang Z. Stress impairs consolidation of recognition memory after blocking drug memory reconsolidation. Neurosci Lett. 2011;501:50-4.

41. Baker KB, Kim JJ. Effects of stress and hippocampal NMDA recepto antagonism on recognition memory in rats. Learn Mem. 2002;9:58-65.

42. González-López MR, García-Saldívar NL, González-Sánchez DJ, Gascón-Enríquez O, Romero-Guadiana JC, Cruz-Morales SE. Efectos de estrés agudo sobre la memoria declarativa y la ansiedad en ratas: efectos de género 2017; LX Congreso Nacional de Ciencias Fisiológicas Nuevo León, Monterrey, México Abstract.

43. Sutcliffe JS, Marshall KM, Neill JC. Influence of gender on working and spatial memory in the novel object recognition task in the rat. Behav Brain Res. 2007; 177:117-25.

44. Harro $\mathrm{H}$. Animals, anxiety, and anxiety disorders: How to measure anxiety in rodents and why. Behav Brain Res. 2018;352:81-93.

45. Lamprea MR, Cardenas FP, Setem J, Morato S. Thigmotatic responses in an open-field. Braz J Med Biol Res. 2008;41:135-40.
46. Woodson JC, Macintosh D, Fleshner M, Diamond DM. Emotion-induced amnesia in rats: working memory-specific impairment, corticosterone-memory correlation, and fear versus arousal effects on memory. Learn Mem. 2003:10:326-36

47. Harris AP, Holmes MC, de Kloet ER, Chapman KE, Seckle JR. Mineralocorticoid and glucocorticoid receptor balance in control of HPA axis and behaviour. Psychoneuroendocrinology. 2013;38:648-58.

48. Schulz K, Korz V. Emotional and cognitive information processing: Relations to behavioral performance and hippocampal long-term potentiation in vivo during a spatial water maze training in rats. Learn Mem. 2010; 17:552-60.

49. Muñoz-Abellán C, Rabasa, Daviu N, Nadal R, Armario A. Behavioral and endocrine consequences of simultaneous exposure to two different stressors in rats: Interaction or independence? Plos One. 2011;6(6):e21426.

50. Bevins RA, Besheer J, Palmatie MI, Jensen HC, Pickett KS, Eurek S. Novel-object place conditioning: Behavioral and dopaminergic processes in expression of novelty reward. Behav Brain Res. 2002;129:41-50.

51. Okuda S, Roozendaal B, McGaugh JL. Glucocorticoid effects on object recognition memory require training-associated emotional arousal. Proc Natl Acad Sci U S A. 2004;101(3):853-8. 